\title{
iTRAQ-based quantitative proteomics analysis of the hepatoprotective effect of melatonin on ANIT-induced cholestasis in rats
}

\author{
DINGNAN WANG ${ }^{1 *}$, HAN YU $^{1,2^{*}}$, YUNZHOU LI $^{1 *}$, ZONGYING XU $^{1}$, SHAOHUA SHI $^{1}$, DOU DOU ${ }^{1}$, \\ LILI SUN $^{1}$, ZHILI ZHENG ${ }^{3}$, XINGHUA SHI $^{3}$, XIULAN DENG $^{3}$ and XIANGGEN ZHONG ${ }^{1}$ \\ ${ }^{1}$ Synopsis of Golden Chamber Department, Chinese Medicine College, Beijing University of \\ Chinese Medicine, Beijing 100029; ${ }^{2}$ Formulas of Chinese Medicine, Basic Medical College of \\ Chengdu University of Traditional Chinese Medicine, Chengdu, Sichuan 611137; ${ }^{3}$ Department of Pharmacology, \\ Chinese Medicine College, Beijing University of Chinese Medicine, Beijing 100029, P.R. China
}

Received October 4, 2019; Accepted April 28, 2021

DOI: $10.3892 /$ etm.2021.10446

\begin{abstract}
The therapeutic effects of melatonin on cholestatic liver injury have received widespread attention recently. The aim of the present study was to investigate the mechanisms of the anti-cholestatic effects of melatonin against $\alpha$-naphthyl isothiocyanate (ANIT)-induced liver injury in rats and to screen for potential biomarkers of cholestasis through isobaric tags for relative and absolute quantitation (iTRAQ) proteomics. Rats orally received melatonin ( $100 \mathrm{mg} / \mathrm{kg}$ body weight) or an equivalent volume of $0.25 \%$ carboxymethyl cellulose sodium
\end{abstract}

Correspondence to: Professor Xianggen Zhong, Synopsis of Golden Chamber Department, Chinese Medicine College, Beijing University of Chinese Medicine, 11 North 3rd Ring East Road, Chaoyang, Beijing 100029, P.R. China

E-mail: zhongxg@bucm.edu.cn

Professor Xiulan Deng, Department of Pharmacology, Chinese Medicine College, Beijing University of Chinese Medicine, 11 North 3rd Ring East Road, Chaoyang, Beijing 100029, P.R. China

E-mail: dengxiulan@bucm.edu.cn

*Contributed equally

Abbreviations: ALP, alkaline phosphatase; ALT, alanine transaminase; ANIT, $\alpha$-naphthyl isothiocyanate; AST, aspartate transaminase; BP, biological processes; $\mathrm{CC}$, cellular component; CMC, carboxymethyl cellulose sodium salt; DBIL, direct bilirubin; DTT, DL-dithiothreitol; FA, formic acid; GO, Gene Ontology; IA, iodoacetamide; iTRAQ, isobaric tags for relative and absolute quantitation; KEGG, Kyoto Encyclopedia of Genes and Genomes; LC-MS/MS, liquid chromatography-tandem mass spectrometry; MF, molecular function; PPAR, peroxisome proliferator-activated receptor; ROS, reactive oxygen species; TBIL, total bilirubin; TEAB, tetraethyl-ammonium bromide; UDCA, ursodeoxycholic acid

Key words: melatonin, cholestasis, proteomics, iTRAQ, ANIT salt $12 \mathrm{~h}$ after intraperitoneal injection of ANIT $(75 \mathrm{mg} / \mathrm{kg})$ and were subsequently sacrificed at $36 \mathrm{~h}$ after injection. Liver biochemical indices were determined and liver tissue samples were stained using hematoxylin-eosin staining, followed by iTRAQ quantitative proteomics to identify potential underlying therapeutic mechanisms and biomarkers. The results suggested that the expression levels of alanine transaminase, aspartate aminotransferase, total bilirubin and direct bilirubin were reduced in the rats treated with melatonin. Histopathological observation indicated that melatonin was effective in the treatment of ANIT-induced cholestasis. iTRAQ proteomics results suggested that melatonin-mediated reduction in ANIT-induced cholestasis may be associated with enhanced antioxidant function and relieving abnormal fatty acid metabolism. According to pathway enrichment analysis using the Kyoto Encyclopedia of Genes and Genomes, the major metabolic pathways for the metabolism of melatonin are fatty acid degradation, the peroxisome proliferator-activated receptor signaling pathway, fatty acid metabolism, chemical carcinogenesis, carbon metabolism, pyruvate metabolism, fatty acid biosynthesis and retinol metabolism, as well as drug metabolism via cytochrome P450. Malate dehydrogenase 1 and glutathione S-transferase $\mathrm{Yb}-3$ may serve as potential targets in the treatment of ANIT-induced cholestasis with melatonin.

\section{Introduction}

Cholestasis is a disease in which bile flow is impaired. Cholestasis may be caused by either intrahepatic or extrahepatic dysfunction (1), and may lead to a range of clinical hepatobiliary diseases, such as liver failure, hepatobiliary malignancy and bile fibrosis, cirrhosis (2). Ursodeoxycholic acid (UDCA) is currently the only treatment approved by the US Food and Drug Administration for the treatment of patients with cholestasis (3). However, according to the Primary Biliary Cholangitis Treatment and Management Guidelines from the British Society of Gastroenterology from 2018, oral UDCA is not an ideal therapeutic option as it may aggravate liver injury and one-third of patients are unresponsive to it $(4,5)$. 
Melatonin is a hormone secreted primarily by the pineal gland (6,7). Melatonin is involved in the regulation of circadian rhythms, such as the sleep/wake rhythm, neuroendocrine rhythm and body temperature cycles $(8,9)$. There are numerous studies suggesting that melatonin may be used to treat liver disease and the hepatoprotective effects of melatonin may be associated with its antioxidant properties (10). Previous studies by our group demonstrated that the therapeutic effects of melatonin against $\alpha$-naphthyl isothiocyanate (ANIT)-induced acute cholestasis are associated with the attenuation of oxidative stress $(11,12)$.

ANIT is a widely utilized chemical substance able to induce acute cholestasis by injuring bile duct epithelium and hepatic parenchymal cells $(13,14)$. However, the mechanisms underlying ANIT-induced acute cholestasis have remained to be fully elucidated. Due to its similarities with drug-induced cholangiolitic hepatitis in humans, ANIT-induced acute cholestasis has been widely used as a model of acute cholestasis in susceptible species, such as rats (15-17).

Proteomics provides essential information on the quantity, function and relationship of protein complexes (18). Isobaric tags for relative and absolute quantitation (iTRAQ) combined with liquid chromatography-tandem mass spectrometry (LC-MS/MS) analysis has been used to determine protein quantities and their compositions (19-22). In the present study, the anti-cholestatic effect of melatonin in rats was assessed using iTRAQ combined with LC-MS/MS to identify the differentially expressed proteins in the samples. The differentially expressed proteins were subjected to Gene Ontology (GO) annotation and Kyoto Encyclopedia of Genes and Genomes (KEGG) analyses in order to understand their effects and mechanisms.

\section{Materials and methods}

Chemicals and drugs. Table I provides details of chemicals and drugs. AST, ALT, TBIL and DBIL were detected by chemical oxidation assays. All other reagents were of analytical grade or higher.

Experimental animals. Male Sprague-Dawley rats (weight, 240-280 g; age, 7 weeks) were procured from SPF-JD-SPF Biotech Co., Ltd. (Beijing, China; certification no. SCXK-JING 2016-0002) and were allowed to acclimatize for 1 week. All rats were maintained in cages with free access to rat chow and water in a temperature-controlled $\left(22-24^{\circ} \mathrm{C}\right)$ environment under a 12-h dark/light cycle with $50 \%$ humidity. The present study was performed in accordance with the guidelines of the animal care regulations of Beijing University of Chinese Medicine and was approved by the Ethics Committee for Animal Care and Treatment of Beijing University of Chinese Medicine (Beijing, China; approval no. bucm-4-2017122735-4035).

Experimental design and experimental groups. Table II provides details on the grouping of the 30 rats. They were randomly allocated into three groups of 10 as follows: Control group, model group and melatonin group. The rats were fasted for $12 \mathrm{~h}$ prior to injections. ANIT was dissolved in olive oil for administration at a dose of $75 \mathrm{mg} / \mathrm{kg}$ body weight; in other words, $1 \mathrm{ml}$ of ANIT solution in olive oil $(75 \mathrm{mg} / \mathrm{ml})$ was given per $100 \mathrm{~g}$ b.w. ANIT solution was administered by intraperitoneal injection at a dose of $75 \mathrm{mg} / \mathrm{kg}$ body weight to induce liver injury with cholestasis in the model and melatonin groups. The control group was injected with an equivalent volume of olive oil $(23,24)$. A total of $12 \mathrm{~h}$ after the ANIT injection, each rat was weighed and intervention was performed according to the group: In the melatonin group, rats were administered melatonin via oral gavage at a dose of $100 \mathrm{mg} / \mathrm{kg}$, whereas the control and model groups were administered with an equivalent volume of $1 \mathrm{ml} 0.25 \% \mathrm{CMC}$. At $36 \mathrm{~h}$ after ANIT treatment, rats were euthanized under pentobarbital anesthesia $(100 \mathrm{mg} / \mathrm{kg}$ ) by exsanguination, and liver tissues and serum samples were taken and frozen rapidly in liquid nitrogen (24). All the samples were stored at $-80^{\circ} \mathrm{C}$.

Serum biochemistry. ALT, AST, TBIL and DBIL levels in rat serum were measured using the corresponding commercially available kits according to the manufacturer's protocol.

$H \& E$ staining and liver biochemistry analysis. After sacrificing the rats, liver tissues were collected and fixed using $10 \%$ formaldehyde and cut into $1 \times 1 \times 0.3 \mathrm{~cm}$ slices. Sections were dehydrated in a gradient of alcohol solutions, embedded in paraffin and the wax blocks were sliced into 4-5 $\mu \mathrm{m}$ sections. All sections were stained with H\&E for analysis of necrosis using a light microscope (BX53 microscope; Olympus Corp.).

Proteomics chemicals and reagents. Urea, DL-dithiothreitol (DTT), iodoacetamide (IA), IPG buffer and formic acid (FA) were purchased from GE Healthcare. SDS, Tris-(hydro xymethyl)-amino-methane, trichloroacetic acid, ammonium persulfate and N,N,N',N'-tetramethylethylenediamine were purchased from Amresco. Tetraethyl-ammonium bromide (TEAB) and Coomassie brilliant blue G-250 were obtained from Sigma-Aldrich (Merck KGaA). Trypsin was from Promega Corp. and acetonitrile (ACN; HPLC grade) and $\mathrm{H}_{2} \mathrm{O}$ were purchased from Thermo Fisher Scientific, Inc.

Protein extraction. Samples were immediately ground in liquid nitrogen and subsequently, $300 \mu$ l SDS lysis buffer was added. Protease inhibitor (phenylmethylsulfonylfluoride) was added to a final concentration of $1 \mathrm{mM}$, followed by mixing. The samples were sonicated in an ice bath at $80 \mathrm{~W}$ with 1-sec on/off cycles for $3 \mathrm{~min}$, which was repeated three times. The supernatant was collected after centrifugation at $12,000 \mathrm{x} g$ for $20 \mathrm{~min}$ at $4^{\circ} \mathrm{C}$. The concentration of extracted protein in the supernatant was determined using a bicinchoninic acid assay. The protein extract was stored at $-80^{\circ} \mathrm{C}$.

Protein digestion and iTRAQ labeling. A total of $100 \mu \mathrm{g}$ protein extract was mixed in $120 \mu \mathrm{l}$ reducing buffer [10 mM DTT, $8 \mathrm{mM}$ urea and $100 \mathrm{mM}$ TEAB, $\mathrm{pH}$ 8.0] and the solution was incubated at $60^{\circ} \mathrm{C}$ for $1 \mathrm{~h}$. IA was added to a final concentration of $50 \mathrm{mM}$ and left in the dark at room temperature for $40 \mathrm{~min}$. The supernatant was collected after centrifugation at $12,000 \mathrm{x}$ g for $20 \mathrm{~min}$ at $4^{\circ} \mathrm{C}$, and $100 \mu 1100 \mathrm{mM}$ TEAB was added, followed by centrifugation at $12,000 \mathrm{x} \mathrm{g}$ for $20 \mathrm{~min}$ at $4^{\circ} \mathrm{C}$ and the supernatant was collected. This step was repeated twice, and after a final centrifugation at 12,000 $\mathrm{x}$ g for $20 \mathrm{~min}$ at $4^{\circ} \mathrm{C}$ and the supernatant was collected, the peptides were collected. A total of $50 \mu 1100 \mathrm{mM}$ TEAB was added, the 
Table I. Details of chemicals and drugs.

\begin{tabular}{ll}
\hline Chemicals/drugs & Company \\
\hline$\alpha$-naphthyl isothiocyanate & Sigma-Aldrich (Merck KGaA) \\
Melatonin & Sigma-Aldrich (Merck KGaA) \\
Carboxymethyl cellulose sodium salt & Yuanye Biological Technology Co., Ltd. \\
Aspartate aminotransferase (cat. no. C010-2) & Nanjing Jiancheng Bioengineering Institute \\
Alanine aminotransferase (cat. no. C0009-2) & Nanjing Jiancheng Bioengineering Institute \\
Total bilirubin (cat. no. C019-1) & Nanjing Jiancheng Bioengineering Institute \\
Direct bilirubin (cat. no. C019-2) & Nanjing Jiancheng Bioengineering Institute
\end{tabular}

Table II. Details of the animal experimental design.

Treatment at each time-point (h)

\begin{tabular}{llllll}
\cline { 2 - 5 } Group & 0 & 12 & 24 & 36 & 48 \\
\hline Control & Fasting & Olive oil & $0.25 \%$ CMC & Fasting & Sacrifice \\
Model & Fasting & $75 \mathrm{mg} / \mathrm{kg} \mathrm{ANIT}$ & $0.25 \% \mathrm{CMC}$ & Fasting & Sacrifice \\
MT & Fasting & $75 \mathrm{mg} / \mathrm{kg} \mathrm{ANIT}$ & $100 \mathrm{mg} / \mathrm{kg} \mathrm{MT}$ & Fasting & Sacrifice \\
\hline
\end{tabular}

MT, melatonin; ANIT, $\alpha$-naphthyl isothiocyanate; CMC, carboxymethyl cellulose sodium salt.

mixture was centrifuged at $12,000 \mathrm{x}$ g for $20 \mathrm{~min}$ at $4^{\circ} \mathrm{C}$ again and the supernatant was collected and lyophilized. The sample was reconstituted in $100 \mu 1100 \mathrm{mM}$ TEAB and subsequently, $40 \mu 1$ sample was transferred to a new tube for labeling. A total of $100 \mu \mathrm{l}$ of iTRAQ reagent was transferred to the sample tube and $200 \mu \mathrm{l}$ water was added to quench the labeling reaction. The solution was lyophilized and the sample was stored at $-80^{\circ} \mathrm{C}$.

MS analysis. All analyses were performed on a Triple time of flight $5600 \mathrm{MS}$ machine (SCIEX) equipped with a Nanospray III source (SCIEX). Samples were loaded onto a capillary C18 trap column ( $3 \mathrm{~cm} \times 100 \mu \mathrm{m})$ and then separated by a C18 column $(15 \mathrm{~cm} \times 75 \mu \mathrm{m})$ on an Eksigent nanoLC-1D plus system (SCIEX). The flow rate was $300 \mathrm{nl} / \mathrm{min}$ and a linear gradient was applied over 90 min (from 5-85\% B over 90 min; mobile phase A was $0.1 \%$ FA in water and phase B was $95 \% \mathrm{ACN} / 0.1 \% \mathrm{FA}$ in water). Data were acquired using a 2.4-kV ion spray voltage, 35 psi curtain gas, 5 psi nebulizer gas and an interface heater temperature at $150^{\circ} \mathrm{C}$. The MS scanned between 400 and $1,500 \mathrm{~m} / \mathrm{z}$ with an accumulation time of $250 \mathrm{msec}$. For Information-Dependent Acquisition, $30 \mathrm{MS} / \mathrm{MS}$ spectra ( $80 \mathrm{msec}$ each; mass range, $100-1,500 \mathrm{~m} / \mathrm{z}$ ) of $\mathrm{MS}$ peaks above an intensity of 260 with a charge state between 2 and 5 were acquired. A rolling collision energy voltage was used for collision induced dissociation fragmentation and MS/MS spectra acquisitions. Mass was dynamically excluded for $22 \mathrm{sec}$.

Bioinformatics analysis of proteomic data. Functional analysis of the differentially expressed proteins identified was performed using GO annotation in the categories molecular function (MF), biological process (BP) or cellular component
(CC) with the Database for Annotation, Visualization and Integrated Discovery (DAVID) version 6.8 (david.ncifcrf.gov/). The overall steps consisted of Sequence alignment, GO entry extraction mapping, GO annotation and data augmentation. Subsequently, KEGG (genome.jp/kegg/pathway.html) was used to predict the metabolic pathways of proteins in cells and the function of these proteins. The data were obtained using the Search Tool for the Retrieval of Interacting Genes and proteins (STRING) network analysis (string-db.org/) and Cytoscape version 3.7.1. To be considered differentially expressed proteins, the criteria were $\mathrm{P}<0.05$ and a fold-change $>1.5$ or $<0.67$.

Western blot analysis. Western blots were performed using automated capillary western blot, an automated capillary-based size sorting system (ProteinSimple). All procedures were performed according to the manufacturer's protocol. In brief, $8 \mu \mathrm{g}$ of diluted protein lysate was mixed with $2 \mu \mathrm{g}$ fluorescent Master Mix (mixed 5 times) and heated at $95^{\circ} \mathrm{C}$ for $5 \mathrm{~min}$. The samples, blocking reagents, wash buffer, primary antibodies, secondary antibodies and chemiluminescent substrate were dispensed into designated wells in a manufacturer-provided microplate. The plate was loaded into the instrument and protein was drawn into individual capillaries using a 25-capillary cassette, provided by the manufacturer. Protein separation and obtainment of the resulting chemiluminescent signal were performed automatically on the individual capillaries using the default settings. The data were analyzed using Compass software (ProteinSimple; v2.7.1). Malate dehydrogenase 1 (MDH1) and glutathione S-transferase Yb-3 (GSTM3) antibodies were purchased from ProteinTech (1:50 dilution; cat. no. 15904-1-AP and 15214-1-AP, respectively); $\beta$-actin antibody was obtained from Cell Signaling Technology, Inc. (1:50 dilution; cat. no. 4970; Cell Signaling Technology, 
A

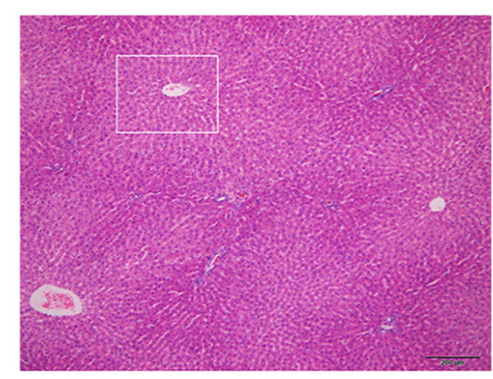

D

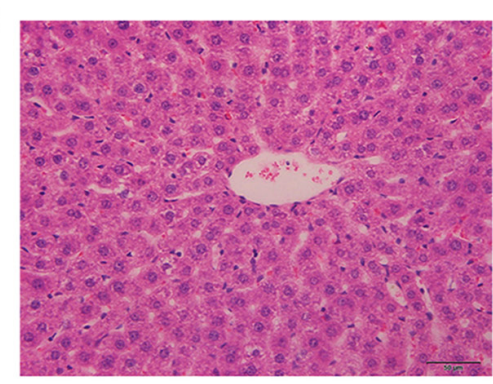

B

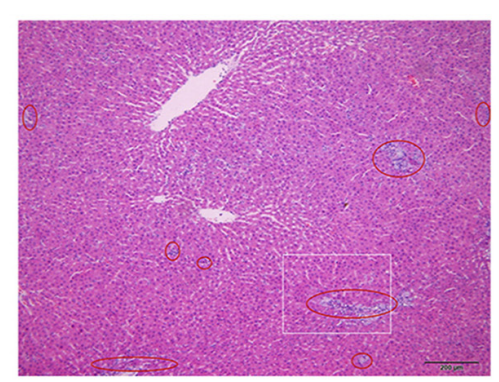

E

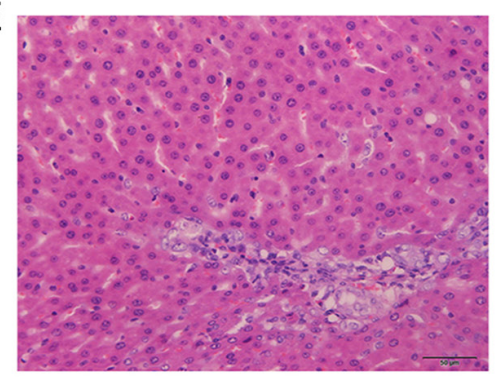

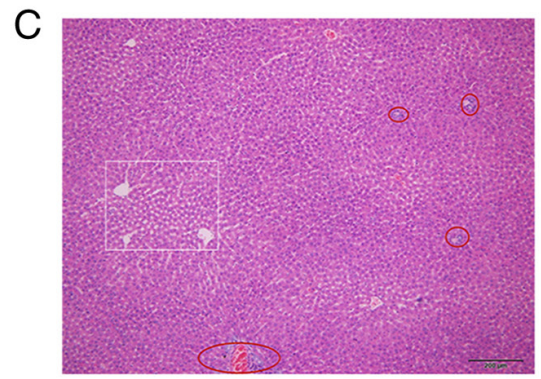

$\mathrm{F}$

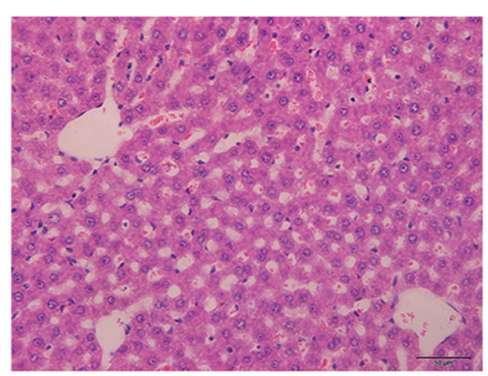

G

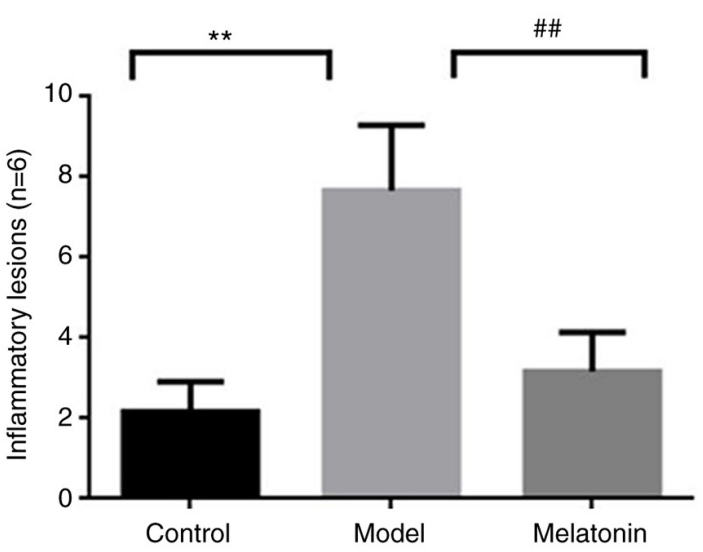

Figure 1. Melatonin reverses histological damage in the liver tissues of rats with $\alpha$-naphthyl isothiocyanate-induced cholestasis. Histology images of (A) Control group, (B) cholestasis model group and (C) Melatonin treatment group (magnification, x50; scale bar, $200 \mu \mathrm{m}$; H\&E staining. Liver tissue damage is indicated by red circles). (D-F) Excerpts from magnified windows from A-C, respectively. (D) Control group, (E) cholestasis model group and (F) Melatonin treatment group (magnification, x200; scale bar, $50 \mu \mathrm{m}$ ). (G) Quantitative results of inflammatory lesions observed from histology images. Values are expressed as the mean \pm standard error of the mean $\left(\mathrm{n}=6\right.$ per group). ${ }^{* *} \mathrm{P}<0.01 ;{ }^{\# \#} \mathrm{P}<0.01$.

Inc.) and used as the loading control. Secondary antibody was purchased from ProteinSimple (cat. no. 042-206). Incubation with primary and secondary antibodies was performed at room temperature for $30 \mathrm{~min}$.

Statistical analysis. Data analyses were performed using GraphPad Prism software (version 5.0; GraphPad Software, Inc.). The individual statistical tests used were the Mann-Whitney U-test, Wilcoxon's matched-pairs signed-rank test and Spearman's test for correlations. Student's t-test was used for analysis of statistical significance between two groups and one-way analysis of variance followed by Dunnett's post-hoc test was applied to analyse statistical significance among three or more groups. $\mathrm{P}<0.05$ was considered to indicate a statistically significant difference.

\section{Results}

Histological observations. The histology results are provided in Fig. 1. H\&E staining of the control group indicated a normal hepatic lobular structure with hepatic cell cords arranged radially outward from the terminal venules, as well as a uniformly distributed portal area (Fig. 1D). The specimens from the ANIT-induced model group displayed evidence of neutrophil infiltration in the bile duct between the hepatic lobule, sinusoid congestion and necrotic inflammation (Fig. 1E). A small amount of neutrophil infiltration was observed in the melatonin-treated group, with mild inflammation and mild cell edema (Fig. 1F). These results were consistent with those of previous studies by our group $(11,12)$. For each group, 6 random fields of view were statistically evaluated for inflammatory lesions (examples in Fig. 1A-C) and the quantitative results are presented in Fig. 1G. The quantitative results indicated that a significant amount of inflammatory lesions appeared in the model vs. control group, which was significantly decreased in the melatonin vs. model group.

Effects of melatonin on serum biochemistry. The levels of ALT, AST, TBIL and DBIL were significantly increased in the rats intraperitoneally injected with ANIT and were significantly 
A

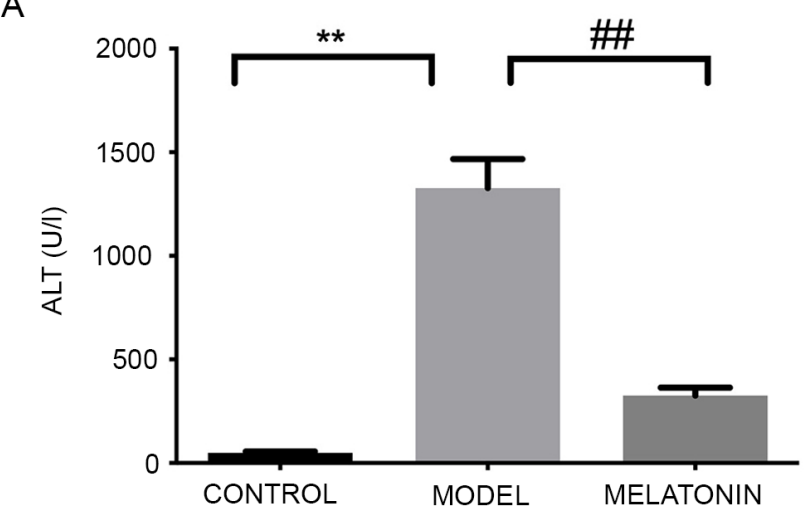

C

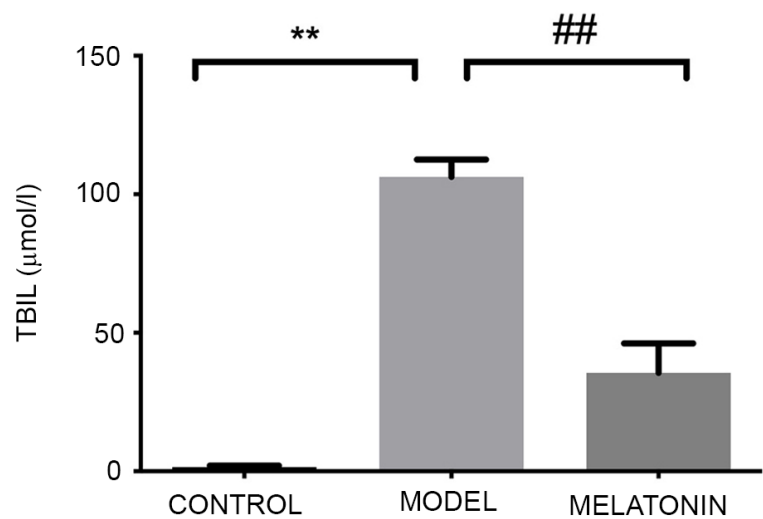

B

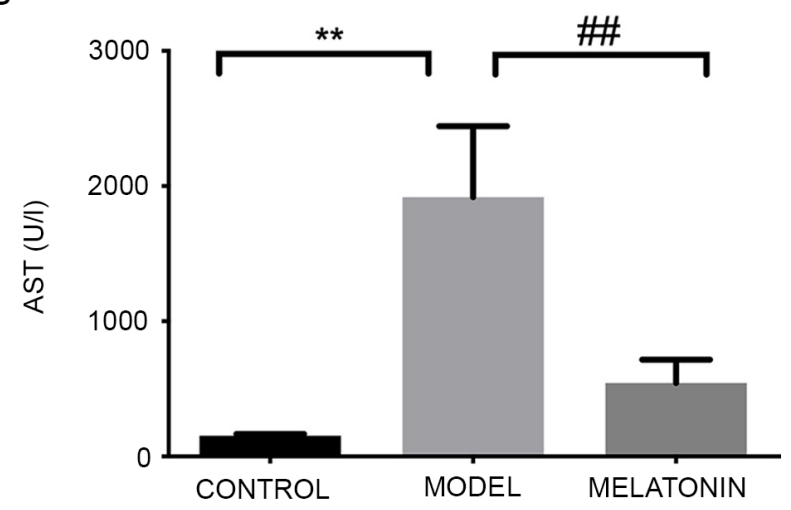

D

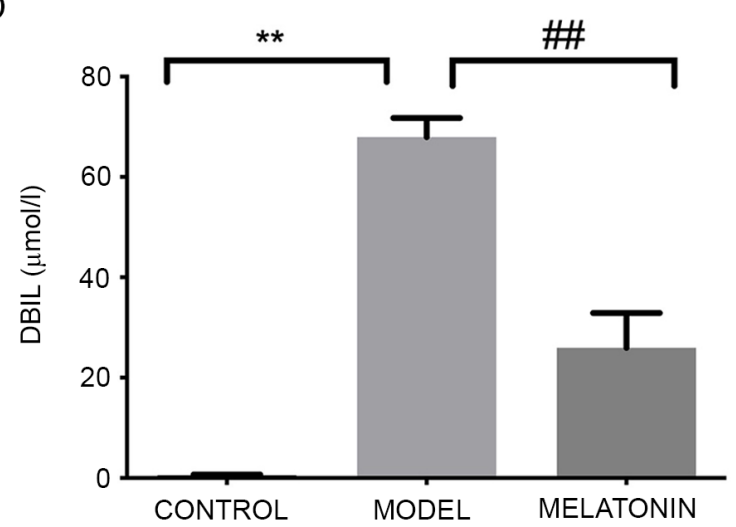

Figure 2. Protective effects of melatonin on cholestasis and hepatotoxicity induced by alpha-naphthyl isothiocyanate. (A) ALT, (B) AST, (C) TBIL and (D) DBIL. Values are expressed as the mean \pm standard error of the mean $\left(\mathrm{n}=10\right.$ per group). ${ }^{* *} \mathrm{P}<0.01$; ${ }^{\# \#} \mathrm{P}<0.01$. ALT, alanine transaminase; AST, aspartate transaminase; TBIL, total bilirubin; DBIL, direct bilirubin.

reduced following treatment with melatonin (Fig. 2). These results were consistent with those of previous studies by our group $(11,12)$.

ITRAQ-based quantitative proteomics analysis of the hepatoprotective effect of melatonin. The hepatic tissue proteins from the three groups with technical duplicates were pooled for the iTRAQ coupled LC-MS/MS analysis. A total of 2,059 proteins were detected and quantified with a minimum confidence level of $95 \%$ and all proteins contained 2 or more peptides. After screening the data, 327 dynamically changed proteins were obtained in each different group. There were 63 differentially expressed proteins between control group vs. model group (Model-Control) and model group vs. melatonin group (Melatonin-Model) (Figs. 3 and 4). Therefore, further research should assess the function of these proteins.

Results of classification of differential protein analysis. GO analysis indicated that the functional terms in the BP category were 'small molecule metabolic process', 'organic acid metabolic process', 'oxoacid metabolic process', 'single-organism metabolic process', 'carboxylic acid metabolic process' and 'oxidation-reduction process' (Fig. 5A). In the category $\mathrm{CC}$, the most relevant enriched terms by the differentially expressed proteins were 'mitochondrion', 'extracellular exosome', 'cytosol', 'organelle membrane', 'endoplasmic reticulum membrane' and 'cytoplasm' (Fig. 5B). The most relevant enriched terms in the category MF were 'oxidoreductase activity', catalytic activity, cofactor binding and acting on $\mathrm{CH}-\mathrm{OH}$ group of donors (Fig. 5C).

KEGG pathway analysis indicated that the main pathways enriched by the differentially expressed proteins were 'metabolic pathways', 'fatty acid degradation', 'PPAR signaling pathway', 'fatty acid metabolism', 'chemical carcinogenesis', 'carbon metabolism', 'pyruvate metabolism', 'fatty acid biosynthesis' and 'retinol metabolism' (Fig. 6). A network of the top 10 most significantly enriched pathways, the top 38 most significantly enriched proteins and their interactions is provided in Fig. 7. Table III shows the details of the 63 differentially expressed proteins of $\alpha$-naphthyl isothiocyanate-induced cholestasis rats with or without melatonin treatment.

Western blot analysis. Western blot experiments were performed to validate the results of iTRAQ combined with the LC-MS/MS analysis. The two proteins MDH1 and GSTM3 were selected for this comparison. The results indicated that the ratios of MDH1 and GSTM3 were decreased following intraperitoneal injection of ANIT and increased with melatonin treatment. These results were consistent with the proteomics data (Fig. 8).

\section{Discussion}

The pathogenesis of acute cholestasis has remained to be fully elucidated. Long-term biliary obstruction may cause liver damage, even induce biliary cirrhosis or liver fibrosis and, in 


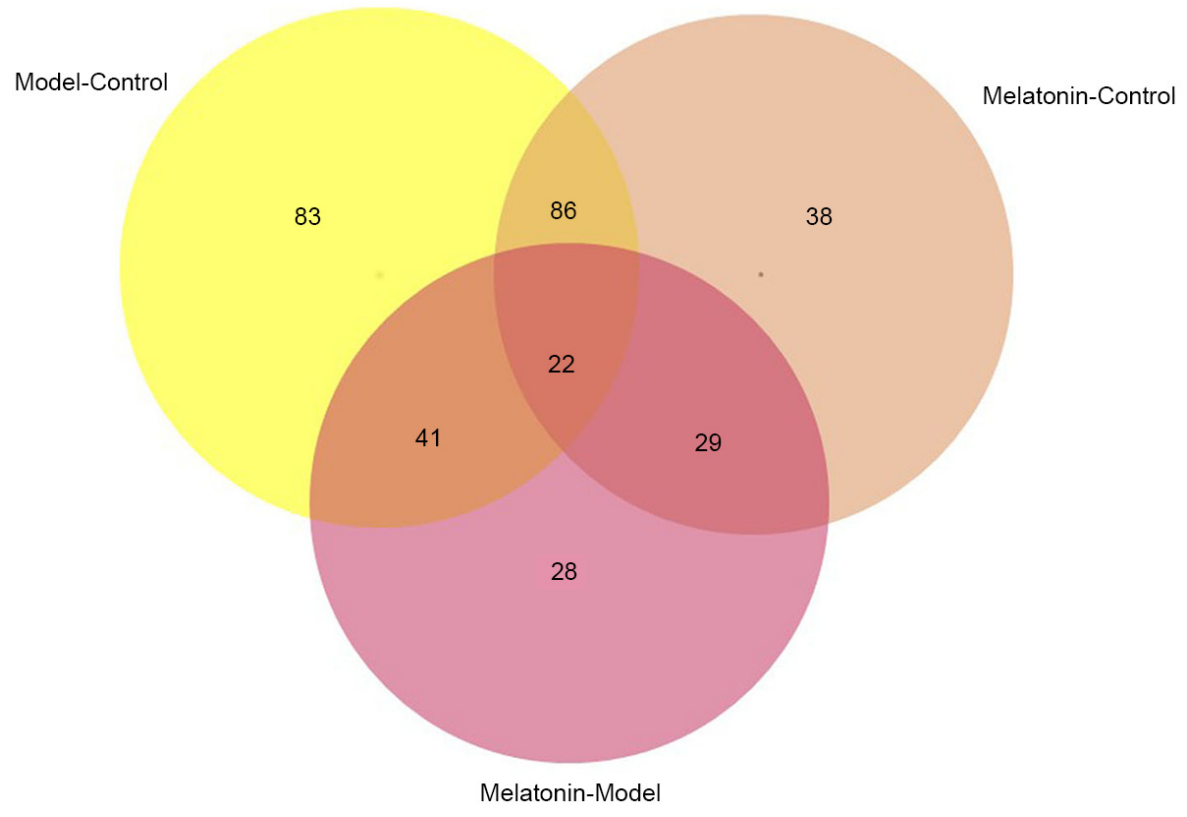

Figure 3. Venn diagram indicating differentially expressed proteins of cholestasis rats with or without melatonin treatment in the model vs. control groups (Model-Control), melatonin vs. control groups (Melatonin-Control) and melatonin vs. model groups (Melatonin-Model).

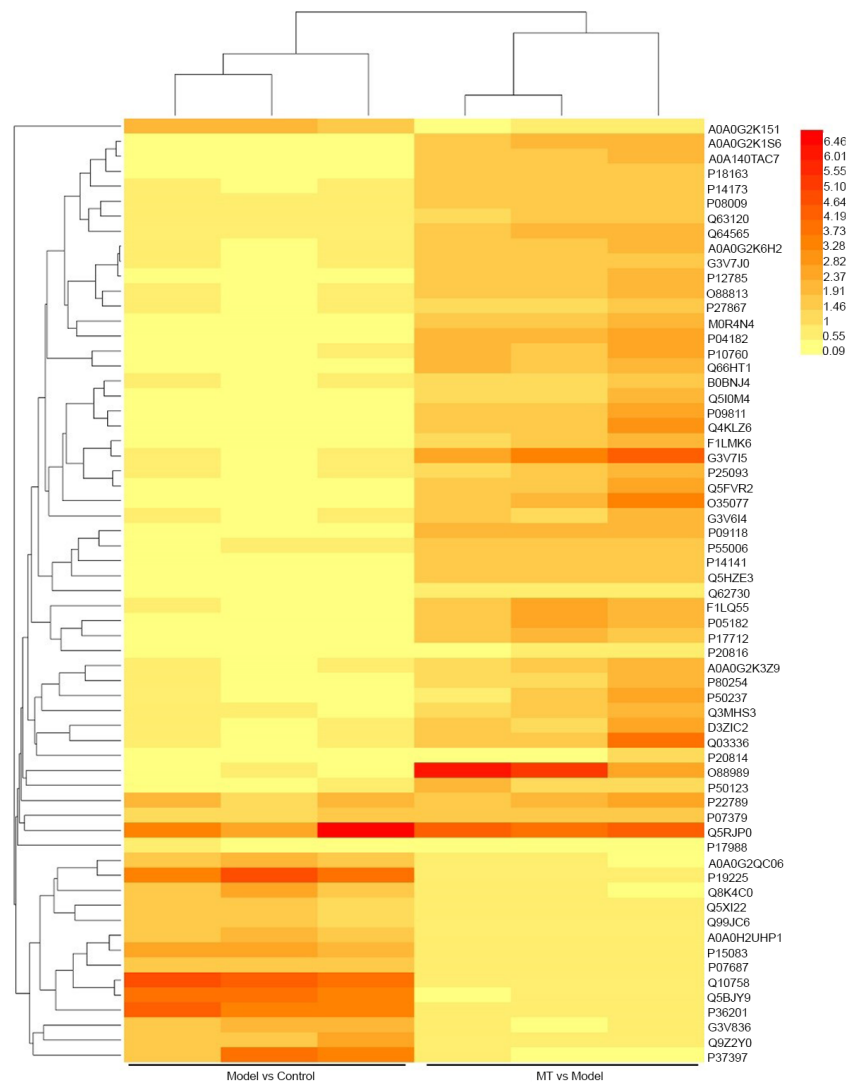

Figure 4. Heatmap diagram indicating the variation tendency of differentially expressed proteins in cholestasis rats with or without melatonin treatment. Their associated Uniprot ID is provided on the right of the heat map. Red indicates upregulation and yellow downregulation. MT, melatonin.

severe cases, may lead to liver failure (25). In recent years, it has been indicated that oxidative stress and abnormal fatty acid metabolism may be involved in the pathogenesis of intrahepatic cholestasis $(11,12,24,26)$. Previously, melatonin has been demonstrated to improve liver fibrosis and liver damage caused by various diseases through inhibiting oxidative damage (10). In the present study, the differential expression protein profile following melatonin treatment for ANIT-induced liver injury in rats was determined using iTRAQ-coupled LC-MS/MS analysis. A total of 63 significantly differentially expressed proteins were identified and two representative proteins were analyzed by western blot based on the iTRAQ results.

The results of the KEGG pathway analysis indicated that the PPAR signaling pathway was associated with fatty acid degradation (Fig. 9), metabolism and biosynthesis. In addition, the PPAR signaling pathway was involved in pyruvate metabolism and citrate cycle, and linked to carbon metabolism by ACAT2, MDH1 and ME1. The PPAR signaling pathway and fatty acid degradation were also associated with retinol metabolism via CYP4A2, and carbon metabolism via CYP2C22, CYP4A2 and CYP2C13. In carbon metabolism, GSTM3 was linked to drug metabolism by cytochrome $\mathrm{P} 450$.

It has been hypothesized that excessive accumulation of bile acids may induce oxidative stress. This may result in an abnormally oxidized state of the internal environment, inducing mitochondrial generation of reactive oxygen species (ROS) by interfering with compounds such as those involved in mitochondrial respiratory chain complexes. These ROS are highly toxic and cause damage to liver cells (24). Melatonin is a mitochondrial targeting antioxidant, which is synthesized in mitochondria, and the mitochondria are also the sites of melatonin metabolism (27). The treatment of liver diseases is constantly being explored and several novel drugs are based on anti-oxidative strategies. They interfere with the pathological mechanisms underlying mitochondrial damage, oxidative stress and ROS production (28-30). Oxidoreductase activity was among the top MF terms enriched by the differentially expressed proteins was. Thus, it was speculated that redox reactions were enhanced, and the imbalance between oxidation 
A

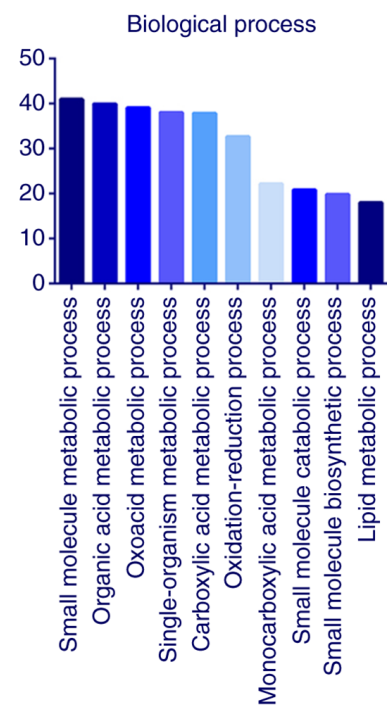

B

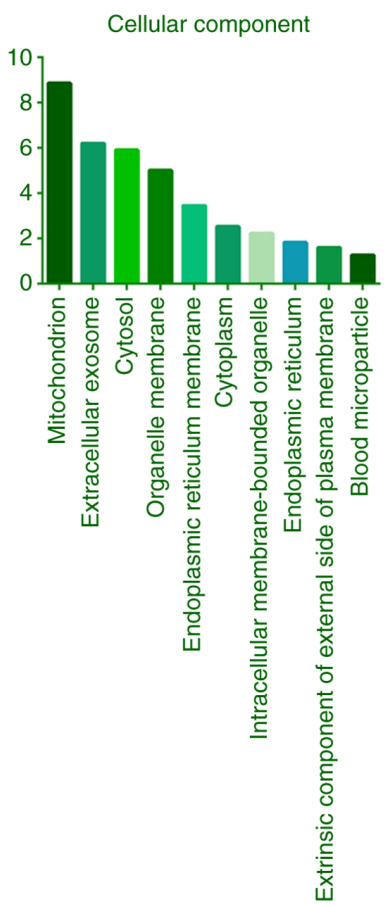

C

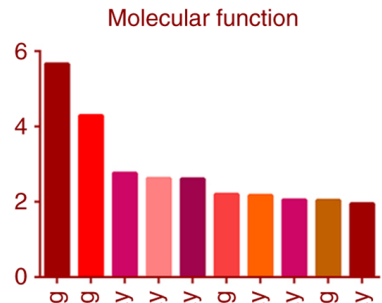

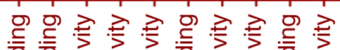

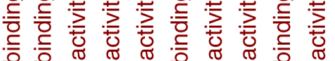

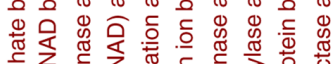

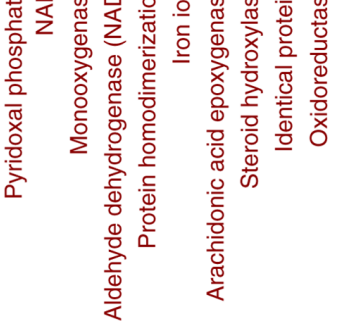

Figure 5. Gene Ontology analysis of the differentially expressed proteins of cholestasis rats with or without melatonin treatment. Functional terms enriched by the proteins were determined in the three major categories (A) biological process, (B) cellular component and (C) molecular function. The y-axis indicates the enrichment ratio of a specific category of proteins in each major category.

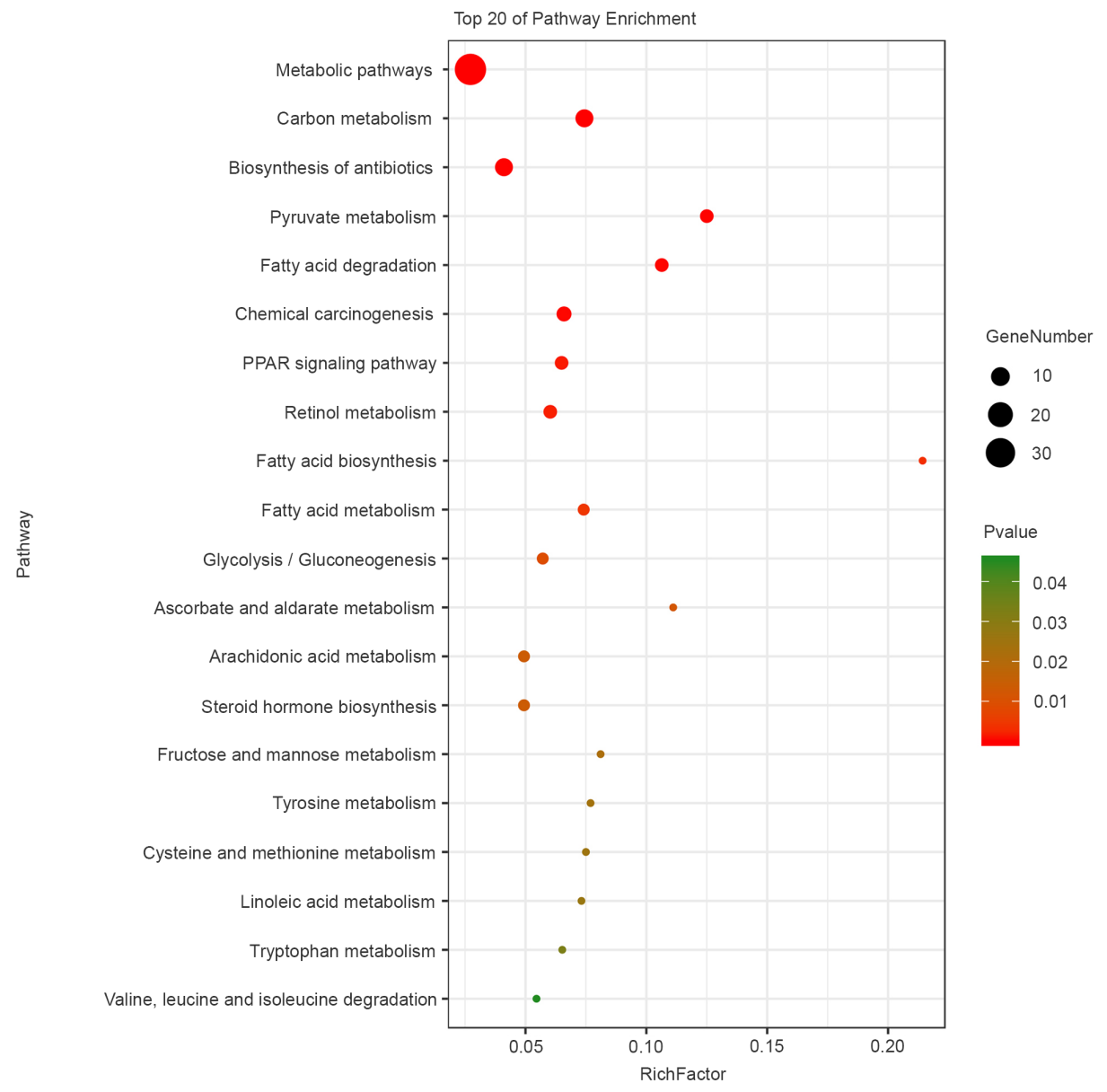

Figure 6. Changes in metabolome profiles in the cholestasis rats with or without melatonin treatment. Bubble chart displaying Kyoto Encyclopedia of Genes and Genomes pathways enriched. The $y$-axis represents the pathways and the $\mathrm{x}$-axis represents the enrichment factor. The color and size of the bubble in the chart represent the enrichment significance and the amount of differentially expressed genes enriched in the pathway, respectively. PPAR, peroxisome proliferator-activated receptor. 


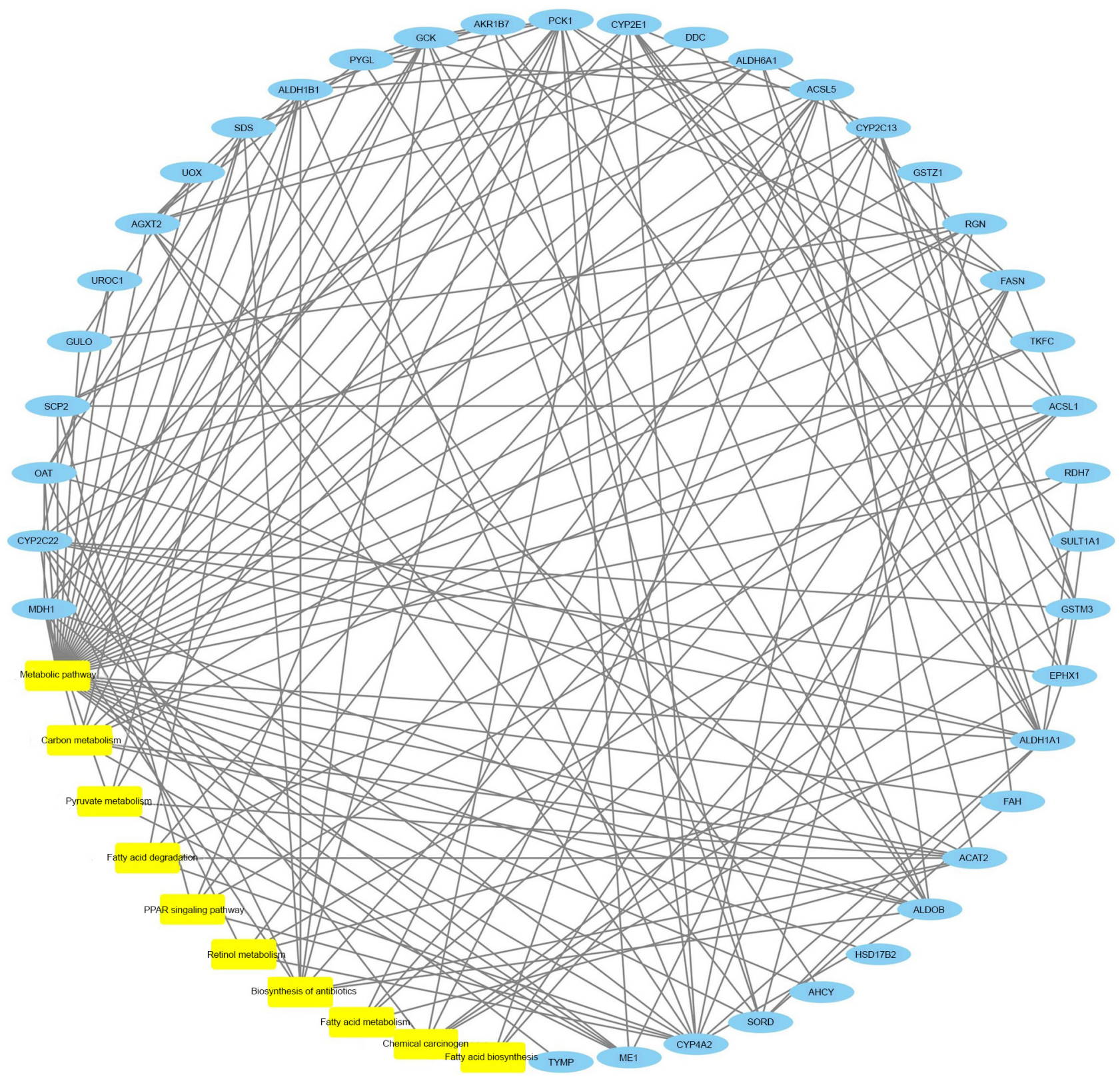

Figure 7. Protein-protein interaction network analysis of the differentially expressed proteins of cholestasis rats with or without melatonin treatment.

and antioxidant systems was reduced following mitochondrial synthesis enhanced by melatonin treatment. Thus, it was hypothesized that the therapeutic mechanism underlying melatonin treatment of acute cholestasis may be associated with the alleviation of oxidative stress by enhancing antioxidant function reducing ROS levels. A previous study by our group indicated that glutathione (GSH) serves a pivotal role in the antioxidant defense in intrahepatic cholestasis. In addition, GSH participates in oxidative defense through its catalysis by glutathione S-transferase (GST) and glutathione peroxidase. GSTM3, a member of the GST family that upregulates the expression of proteins, participates in oxidative defense to repair liver damage (31). In the present study, the expression levels of GSTM3 were increased and the results were consistent with those of the western blot analysis. Thus, GSTM3 may be a potential biomarker for the treatment of intrahepatic cholestasis by melatonin.
Mitochondrial $\beta$-oxidation, peroxisome $\beta$-oxidation, $\omega$-oxidation and microsome pathways are oxidized forms of fatty acids in humans. Among these, $\beta$-oxidation of mitochondrial long-chain fatty acids is the primary pathway of cellular oxidation. $\omega-3$ polyunsaturated fatty acids have been reported to be effective in the prevention and treatment of cholestasis and n-3 polyunsaturated fatty acids may also exhibit therapeutic potential (32). In the present study, KEGG analysis suggested that fatty acid degradation, biosynthesis and metabolism were significantly different prior to and after treatment. Protein lysine acetylation is a type of post-translational modification and acetylated proteins regulate important metabolic processes such as fatty acid metabolism (33). In cells, acetyl-CoA either enters the tricarboxylic acid cycle or is used to synthesize fatty acids. MDH1 catalyzes the conversion of malic acid to oxaloacetate (34). Glucose is known to undergo glycolysis 
Table III. Details of the 63 differentially expressed proteins of $\alpha$-naphthyl isothiocyanate-induced cholestasis rats with or without melatonin treatment.

\begin{tabular}{|c|c|c|c|}
\hline Uniprot ID & Protein name (gene symbol) & F-value & P-value \\
\hline A0A0G2K151 & Apolipoprotein E (APOE) & 0.5889 & 0.0090 \\
\hline A0A0G2K1S6 & Malic enzyme 1 (ME1) & 1.9905 & 0.0150 \\
\hline A0A0G2K3Z9 & $\mathrm{N} / \mathrm{A}$ & - & - \\
\hline A0A0G2K6H2 & Glutathione S-transferase zeta 1 (GSTZ1) & 1.6831 & 0.0295 \\
\hline A0A0G2QC06 & Transferrin (TF) & 0.6179 & 0.0091 \\
\hline A0A0H2UHP1 & Aldehyde dehydrogenase 1 family, member A1 (ALDH1A1) & 0.6223 & 0.0039 \\
\hline A0A140TAC7 & Gulonolactone (L-) oxidase (GULO) & 1.8658 & 0.0162 \\
\hline B0BNJ4 & ETHE1, persulfide dioxygenase (ETHE1) & 1.5354 & 0.0857 \\
\hline D3ZIC2 & Urocanate hydratase 1 (UROC1) & 1.7511 & 0.1896 \\
\hline F1LMK6 & Serine dehydratase (SDS) & 1.7656 & 0.0766 \\
\hline F1LQ55 & Sterol carrier protein $2(\mathrm{SCP} 2)$ & 2.0842 & 0.0795 \\
\hline G3V6I4 & Mitochondrial amidoxime reducing component 1 (MARC1) & 1.5733 & 0.1288 \\
\hline G3V7I5 & Aldehyde dehydrogenase 1 family, member B1 (ALDH1A1) & 3.5411 & 0.0442 \\
\hline G3V7J0 & Aldehyde dehydrogenase 6 family, member A1 (ALDH6A1) & 1.5926 & 0.0348 \\
\hline G3V836 & Clusterin (CLU) & 0.6256 & 0.0209 \\
\hline M0R4N4 & Dehydrogenase/reductase (SDR family) member 7 (DHRS7) & 1.6838 & 0.0487 \\
\hline O35077 & Glycerol-3-phosphate dehydrogenase 1 (GPD1) & 2.4498 & 0.1107 \\
\hline O88813 & Acyl-CoA synthetase long-chain family member 5 (ACSL5) & 1.6460 & 0.0447 \\
\hline O88989 & Malate dehydrogenase 1 (MDH1) & 4.6705 & 0.0673 \\
\hline P04182 & Ornithine aminotransferase (OAT) & 2.3116 & 0.0180 \\
\hline P05182 & Cytochrome P450, family 2, subfamily e, polypeptide 1 (CYP2E1) & 2.1076 & 0.0205 \\
\hline P07379 & Phosphoenolpyruvate carboxykinase 1 (PCK1) & 1.6217 & 0.0094 \\
\hline P07687 & Epoxide hydrolase 1 (EPHX1) & 0.6197 & 0.0010 \\
\hline P08009 & Glutathione S-transferase, mu 3 (GSTM3) & 1.6969 & 0.0045 \\
\hline P09118 & Urate oxidase (UOX) & 2.1557 & 0.0020 \\
\hline P09811 & Phosphorylase, glycogen, liver (PYGl) & 2.1289 & 0.0647 \\
\hline P10760 & Adenosylhomocysteinase (AHCY) & 2.1517 & 0.0252 \\
\hline P12785 & Fatty acid synthase (FASN) & 1.9435 & 0.0444 \\
\hline P14141 & Carbonic anhydrase 3 (CAR3) & 1.5513 & 0.0001 \\
\hline P14173 & Dopa decarboxylase (DDC) & 1.6658 & 0.0224 \\
\hline P15083 & Polymeric immunoglobulin receptor (PIGR) & 0.6010 & 0.0010 \\
\hline P17712 & Glucokinase (GCK) & 1.9240 & 0.0404 \\
\hline P17988 & Sulfotransferase family 1A member 1 (SULT1A1) & 0.4074 & 0.0080 \\
\hline P18162 & Acyl-CoA synthetase long-chain family member 1 (ACSL1) & 1.6682 & 0.0318 \\
\hline P19225 & Cytochrome P450, family 2, subfamily c, polypeptide 22 (CYP2C22) & 0.6401 & 0.0064 \\
\hline P20814 & Cytochrome P450, family 2, subfamily c, polypeptide 13 (CYP2C13) & 0.6094 & 0.2323 \\
\hline P20816 & Cytochrome P450, family 4, subfamily a, polypeptide 2 (CYP4A2) & 0.6297 & 0.0231 \\
\hline P22789 & $\begin{array}{l}\text { Sulfotransferase family 2A, dehydroepiandrosterone (DHEA)-preferring, } \\
\text { member } 6 \text { (SULT2A6) }\end{array}$ & 2.1173 & 0.0255 \\
\hline P25093 & Fumarylacetoacetate hydrolase (FAH) & 1.6438 & 0.0894 \\
\hline P27867 & Sorbitol dehydrogenase (SORD) & 1.5653 & 0.0658 \\
\hline P36201 & Cysteine-rich protein 2 (CRIP2) & 0.6203 & 0.0038 \\
\hline P37397 & Calponin 3 (CNN3) & 0.5362 & 0.0339 \\
\hline P50123 & Glutamyl aminopeptidase (ENPEP) & 1.5400 & 0.1557 \\
\hline P50237 & Sulfotransferase family 1C member 3 (SULTLC3) & 1.8028 & 0.2110 \\
\hline P55006 & Retinol dehydrogenase 7 (RDH7) & 1.5913 & 0.0071 \\
\hline P80254 & D-dopachrome tautomerase (DDT) & 1.5901 & 0.2094 \\
\hline Q03336 & Regucalcin (RGN) & 2.4009 & 0.1904 \\
\hline Q10758 & Keratin 8 (KRT8) & 0.6412 & 0.0021 \\
\hline Q3MHS3 & Aldo-keto reductase family 1, member $\mathrm{C} 1$ (AKR1C1) & 1.7571 & 0.0756 \\
\hline Q4KLZ6 & Triokinase and FMN cyclase (TKFC) & 2.2275 & 0.0849 \\
\hline
\end{tabular}


Table III. Continued.

\begin{tabular}{llcr}
\hline Uniprot ID & \multicolumn{1}{c}{ Protein name (gene symbol) } & F-value & P-value \\
\hline Q5BJY9 & Keratin 18 (KRT18) & 0.5850 & 0.0080 \\
Q5FVR2 & Thymidine phosphorylase (TYMP) & 2.0643 & 0.0869 \\
Q5HZE3 & Thyroid hormone responsive (THRSP) & 1.7476 & 0.0140 \\
Q5I0M4 & Aldo-keto reductase family 1, member C13 (AKR1C13) & 1.5343 & 0.1205 \\
Q5RJP0 & Aldo-keto reductase family 1, member B7 (AKR1B7) & 4.2623 & 0.0012 \\
Q5XI22 & Acetyl-CoA acetyltransferase 2 (ACAT2) & 0.6613 & 0.0034 \\
Q62730 & Hydroxysteroid (17-beta) dehydrogenase 2 (HSD17B2) & 0.6607 & 0.0004 \\
Q62120 & ATP binding cassette subfamily C member 2 (ABCC2) & 1.5017 & 0.0126 \\
Q64565 & Alanine-glyoxylate aminotransferase 2 (AGXT2) & 1.9370 & 0.0044 \\
Q66HT1 & Aldolase, fructose-bisphosphate B (ALDOB) & 1.9087 & 0.0535 \\
Q8K4C0 & Flavin containing monooxygenase 5 (FMO5) & 0.6264 & 0.0156 \\
Q99JC6 & TAP binding protein (TABP) & 0.6588 & 0.0114 \\
Q9Z2Y0 & Glycine-N-acyltransferase-like 2 (GLYATL2) & 0.6144 & 0.0026
\end{tabular}

N/A, not available.

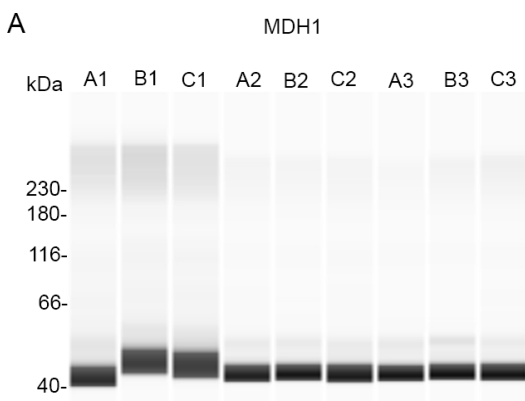

B $\begin{array}{lllllllllll}\mathrm{kDa} & \mathrm{A} 1 & \mathrm{~B} 1 & \mathrm{C} 1 & \mathrm{~A} 2 & \mathrm{~B} 2 & \mathrm{C} 2 & \mathrm{~A} 3 & \mathrm{~B} 3 & \mathrm{C} 3\end{array}$

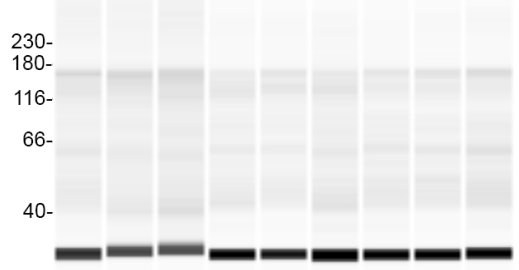

$12-$
C $\quad \beta$-actin $\begin{array}{lllllllllll}\mathrm{kDa} & \mathrm{A} 1 & \mathrm{~B} 1 & \mathrm{C} 1 & \mathrm{~A} 2 & \mathrm{~B} 2 & \mathrm{C} 2 & \mathrm{~A} 3 & \mathrm{~B} 3 & \mathrm{C} 3\end{array}$

$12-$

D

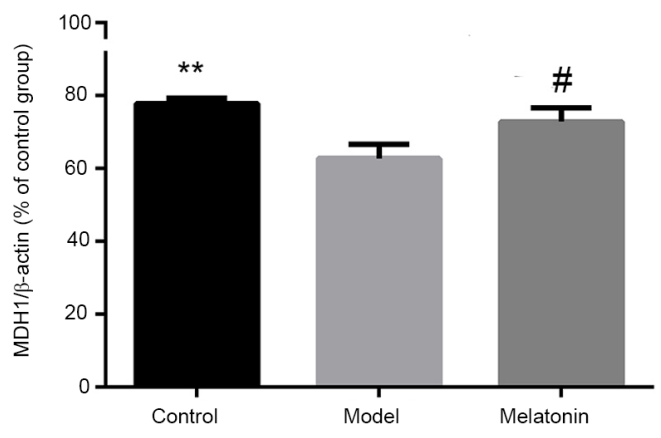

E

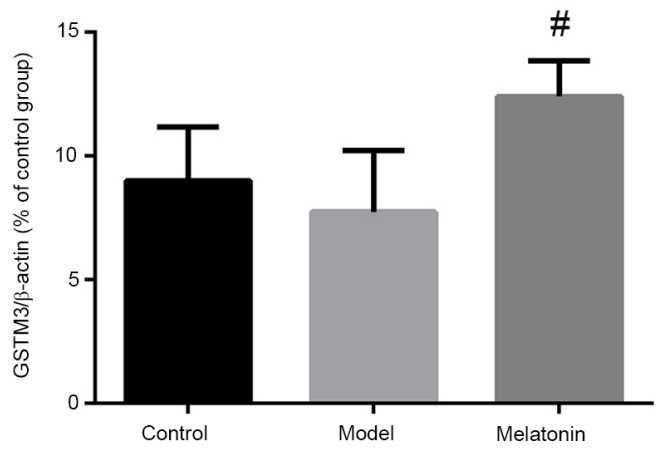

Figure 8. Immunoblotting analysis of GSTM3, MDH1 and $\beta$-actin in the (A) control group, (B) model group and (C) melatonin group. $\beta$-actin was used as the loading control. ( $\mathrm{D}$ and $\mathrm{E}$ ) The levels of protein expression are presented as the mean \pm standard deviation. ${ }^{* *} \mathrm{P}<0.01$ control vs. model group; ${ }^{*} \mathrm{P}<0.05$ melatonin vs. model group. MDH1, malate dehydrogenase 1; GSTM3, glutathione S-transferase Yb-3.

(producing pyruvate), pyruvate subsequently produces acetyl-CoA in the mitochondria and MDH1 promotes the citrate shuttle to provide NADPH and acetyl-CoA for fat synthesis. In the present study, MDH1 was significantly differentially expressed and associated with carbon and pyruvate metabolism. MDH1 is downregulated by ANIT 


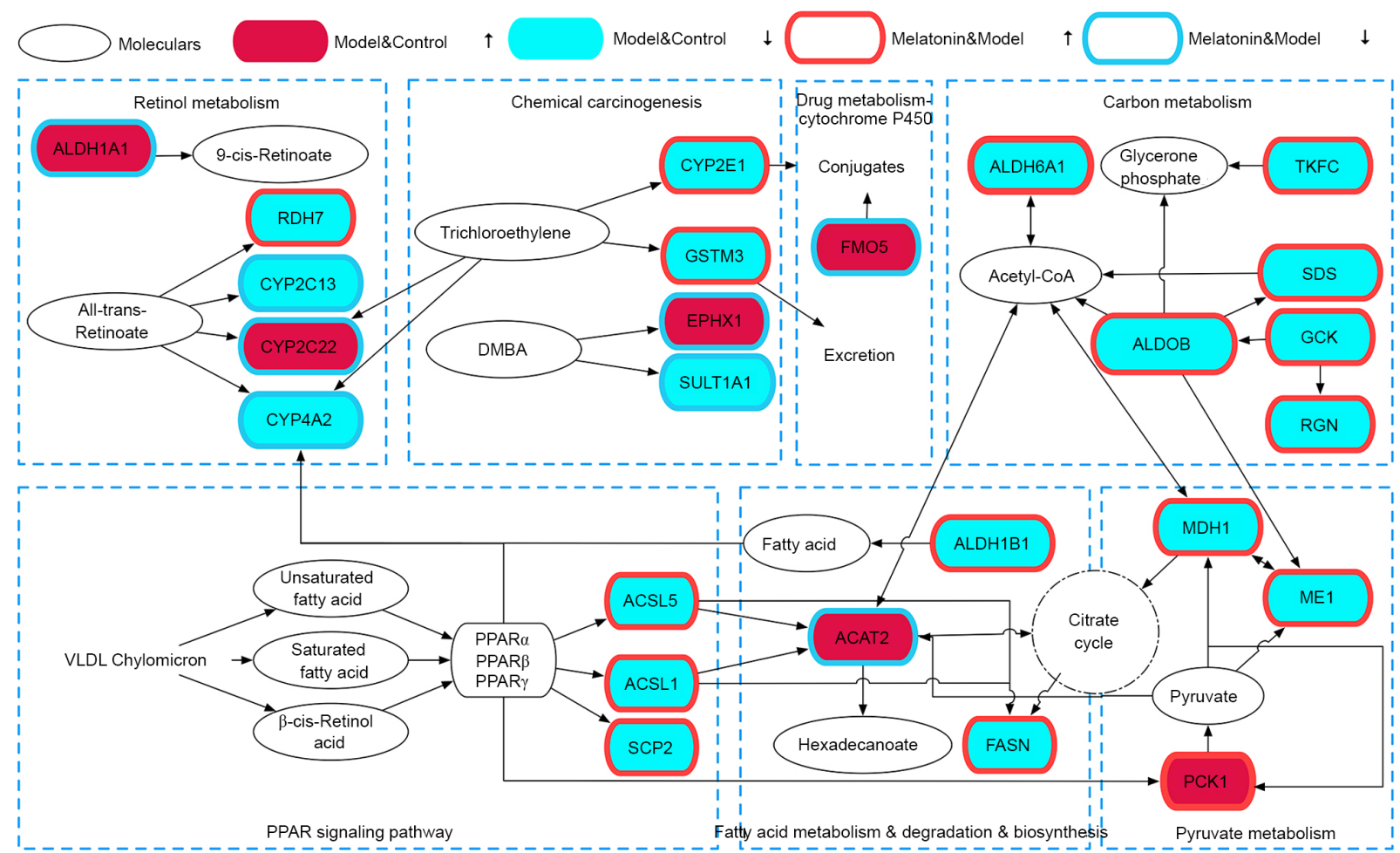

Figure 9. Schematic of 9 significant differential metabolic pathways and the differentially expressed proteins in them of cholestasis rats with or without melatonin treatment. ACAT2, acetyl-CoA acetyltransferase 2; MDH1, malate dehydrogenase 1; ME1, malic enzyme 1; PPAR, peroxisome proliferator-activated receptors; CYP, cytochrome P450; GSTM3, glutathione S-transferase Mu.

and upregulated following melatonin treatment. However, its function in the treatment of cholestasis with melatonin requires further study (35).

The liver is a complex system and its nuclear receptors have the ability to limit bile concentration and siltation by coordinating the stabilization of bile acids and bile secretion (36). One of these nuclear receptors is PPAR. It affects bile balance and cholestatic liver damage in humans (37). The role of PPARs in the liver and fatty acid metabolism primarily include modulation of peroxisome fatty acid oxidation and mitochondrial function (38). The KEGG pathway prediction of the present study suggested that the activity of the PPAR signaling pathway changed significantly between the model vs. control group and returned to near normal in the melatonin group. Therefore, the role of the PPAR signaling pathway in the treatment of cholestasis by melatonin requires further validation.

Cytochrome $\mathrm{P} 450$ is a broad-spectrum biocatalyst with catalytic activity. It primarily occurs in the liver but also in other tissue types and is the primary enzyme involved in melatonin metabolism (39). Cytochrome P450 was hypothesized to be relevant to the physiological production of $\mathrm{O}_{2}$, which may result in oxidative stress under pathological conditions (40). Cytochrome P450 is also involved in the regulation of bile acids and may participate in melatonin metabolism in rat livers (41). Studies have indicated that a lack of cytochrome P450 may affect the metabolism of endogenous substances, such as cholesterol and bile acids (42). In the present study, there was a significant difference in metabolism of xenobiotics by cytochrome $\mathrm{P} 450$ based on the KEGG pathway analysis between the melatonin group and model group. Some of these proteins, such as CYP1A2 and CYP2E1, are known to serve a role in the treatment of liver disease.

In conclusion, the results of the present study suggest that the therapeutic mechanism of melatonin in the treatment of acute cholestasis may be associated with enhancing antioxidant function and relieving abnormal fatty acid metabolism. The primary metabolic pathways of melatonin in the treatment of cholestasis were fatty acid degradation, the PPAR signaling pathway, fatty acid metabolism, chemical carcinogenesis, carbon metabolism, pyruvate metabolism, fatty acid biosynthesis and retinol metabolism, as well as drug metabolism mediated by cytochrome P450. MDH1 and GSTM3 may be potential biomarkers for melatonin treatment of intrahepatic cholestasis in the ANIT-induced animal model. However, these proteomic results are preliminary data and further studies are required to determine the role of these proteins in the treatment of cholestasis with melatonin.

\section{Acknowledgements}

The authors would like to thank Professor Jian Li of the Pathology Department of Beijing University of Chinese Medicine (Beijing, China) and Mrs. Shujing Zhang of the Science Center Department of Beijing University of Chinese Medicine (Beijing, China) for their technical support.

\section{Funding}

The present study was supported by the National Natural Science Foundation of China (grant no. 81573963). 


\section{Availability of data and materials}

The mass spectrometry proteomics data have been deposited to the ProteomeXchange Consortium (http://proteomecentral. proteomexchange.org) via the iProX partner repository with the dataset identifier PXD023155. The data can be accessed online at: https://www.iprox.org/page/project.html?id=IPX0002663000.

\section{Authors' contributions}

$\mathrm{XZ}$ and XD made substantial contributions to the study conception. DW, HY and YL performed most of the experiments. ZX, SS and DD performed histopathological analyses. LS, ZZ and XS participated in data analysis and interpretation. DW and HY confirm the authenticity of all the raw data. All authors have read and approved the final version of this manuscript.

\section{Ethics approval and consent to participate}

The study protocol was in strict accordance with the recommendations of the Guidelines for the Care and Use of Laboratory Anim als of the Ministry of Science and Technology of China and was approved by the Medical and Experimental Animal Ethics Committee of Beijing University of Chinese Medicine (Beijing, China).

\section{Patient consent for publication}

Not applicable.

\section{Competing interests}

The authors declare that they have no competing interests.

\section{References}

1. Meadows V, Kennedy L, Kundu D, Alpini G and Francis H: Bile acid receptor therapeutics effects on chronic liver diseases. Front Med (Lausanne) 7: 15, 2020.

2. Hirschfield GM, Heathcote EJ and Gershwin ME: Pathogenesis of cholestatic liver disease and therapeutic approaches. Gastroenterology 139: 1481-1496, 2010.

3. Black DD, Mack C, Kerkar N, Miloh T, Sundaram SS, Anand R, Gupta A, Alonso E, Arnon R, Bulut P, et al: A prospective trial of withdrawal and reinstitution of ursodeoxycholic acid in pediatric primary sclerosing cholangitis. Hepatol Commun 3: 1482-1495, 2019.

4. Chen HL, Wu SH, Hsu SH, Liou BY, Chen HL and Chang MH: Jaundice revisited: Recent advances in the diagnosis and treatment of inherited cholestatic liver diseases. J Biomed Sci 25: 75 , 2018.

5. Mousa HS, Lleo A, Invernizzi P, Bowlus CL and Gershwin ME: Advances in pharmacotherapy for primary biliary cirrhosis. Expert Opin Pharmacother 16: 633-643, 2015.

6. Lin GJ, Huang SH, Chen SJ, Wang CH, Chang DM and Sytwu HK: Modulation by melatonin of the pathogenesis of inflammatory autoimmune diseases. Int J Mol Sci 14: 11742-11766, 2013.

7. Chen CQ, Fichna J, Bashashati M, Li YY and Storr M: Distribution, function and physiological role of melatonin in the lower gut. World J Gastroenterol 17: 3888-3898, 2011.

8. Huang Y, Xu C, He M, Huang W and Wu K: Saliva cortisol, melatonin levels and circadian rhythm alterations in Chinese primary school children with dyslexia. Medicine (Baltimore) 99 e19098, 2020.

9. Joseph D, Chong NW, Shanks ME, Rosato E, Taub NA, Petersen SA, Symonds ME, Whitehouse WP and Wailoo M: Getting rhythm: How do babies do it?. Arch Dis Child Fetal Neonatal Ed 100: F50-F54, 2015.
10. Zhang JJ, Meng X, Li Y, Zhou Y, Xu DP, Li S and Li HB: Effects of melatonin on liver injuries and diseases. Int J Mol Sci 18: 673, 2017.

11. Li Y, Yu H, Xu Z, Shi S, Wang D, Shi X, Wang Y, Zeng B, Deng H, Deng $X$ and Zhong X: Melatonin ameliorates ANIT-induced cholestasis by activating Nrf2 through a PI3K/Akt-dependent pathway in rats. Mol Med Rep 19: 1185-1193, 2019.

12. Yu H, Li Y, Xu Z, Wang D, Shi S, Deng H, Zeng B, Zheng Z, Sun L, Deng $X$ and Zhong X: Identification of potential biomarkers in cholestasis and the therapeutic effect of melatonin by metabolomics, multivariate data and pathway analyses. Int J Mol Med 42: 2515-2526, 2018.

13. Fang ZZ, Tanaka N, Lu D, Jiang CT, Zhang WH, Zhang C, $\mathrm{Du} \mathrm{Z}, \mathrm{Fu} \mathrm{ZW}$, Gao P, Cao YF, et al: Role of the lipid-regulated NF- $\kappa$ B/IL-6/STAT3 axisinalpha-naphthyl isothiocyanate-induced liver injury. Arch Toxicol 91: 2235-2244, 2017.

14. Dahm LJ and Roth RA: Protection against alpha-naphthylisothiocyanate-induced liver injury by decreased hepatic non-protein sulfhydryl content. Biochem Pharmacol 42: 1181-1188, 1991.

15. Wang BL, Zhang CW, Wang L, Tang KL, Tanaka N, Gonzalez FJ, $\mathrm{Xu}$ Y and Fang ZZ: Lipidomics reveal aryl hydrocarbon receptor (Ahr)-regulated lipid metabolic pathway in alpha-naphthyl isothiocyanate (ANIT)-induced intrahepatic cholestasis. Xenobiotica 49: 591-601, 2019.

16. Tjandra K, Sharkey KA and Swain MG: Progressive development of a Th1-type hepatic cytokine profile in rats with experimental cholangitis. Hepatology 31: 280-290, 2000.

17. Connolly AK, Price SC, Connelly JC and Hinton RH: Early changes in bile duct lining cells and hepatocytes in rats treated with alpha-naphthylisothiocyanate. Toxicol Appl Pharmacol 92: 208-219, 1988

18. Mateos J, Estévez O, González-Fernández Á, Anibarro L, Pallarés Á, Reljic R, Mussá T, Gomes-Maueia C, Nguilichane A, Gallardo JM, et al: Serum proteomics of active tuberculosis patients and contacts reveals unique processes activated during Mycobacterium tuberculosis infection. Sci Rep 10: 3844, 2020.

19. Agafonov DE, Deckert J, Wolf E, Odenwälder P, Bessonov S, Will CL, Urlaub H and Lührmann R: Semiquantitative proteomic analysis of the human spliceosome via a novel two-dimensional gel electrophoresis method. Mol Cell Biol 31: 2667-2682, 2011.

20. Ylhä A, Nättinen J, Aapola U, Mikhailova A, Nykter M, Zhou L, Beuerman R and Uusitalo H: Comparison of iTRAQ and SWATH in a clinical study with multiple time points. Clin Proteomics 15: 24, 2018.

21. Bantscheff M, Schirle M, Sweetman G, Rick J and Kuster B: Quantitative mass spectrometry in proteomics: A critical review. Anal Bioanal Chem 389: 1017-1031, 2007.

22. Bantscheff M,Lemeer S, Savitski MM and Kuster B: Quantitative mass spectrometry in proteomics: Critical review update from 2007 to the present. Anal Bioanal Chem 404: 939-965, 2012.

23. Nakamura T, Ohta Y, Ohashi K, Ikeno K, Watanabe R, Tokunaga $\mathrm{K}$ and Harada N: Protective effect of brazilian propolis against liver damage with cholestasis in rats treated with $\alpha$-Naphthylisothiocyanate. Evid Based Complement Alternat Med 2013: 302720, 2013.

24. Ohta Y, Kongo-Nishimura M, Imai Y and Kitagawa A: Melatonin attenuates disruption of serum cholesterol status in rats with a single alpha-naphthylisothiocyanate treatment. J Pineal Res 42: 159-165, 2007.

25. Chai J, Feng X, Zhang L, Chen S, Cheng Y, He X, Yang Y, He Y, Wang H, Wang R and Chen W: Hepatic expression of detoxification enzymes is decreased in human obstructive cholestasis due to gallstone biliary obstruction. PLoS One 10: $\mathrm{e} 0120055,2015$.

26. Zhang Y, Li F, Patterson AD, Wang Y, Krausz KW, Neale G, Thomas S, Nachagari D, Vogel P, Vore M, et al: Abcb11 deficiency induces cholestasis coupled to impaired $\beta$-fatty acid oxidation in mice. J Biol Chem 287: 24784-24794, 2012.

27. Tan DS and Russel RJ: Mitochondria: The birth place, battle ground and the site of melatonin metabolism in cells. Melatonin Res 2: 44-66, 2019.

28. Chen HH, Chen YT, Yang CC, Chen KH, Sung PH, Chiang HJ, Chen $\mathrm{CH}$, Chua S, Chung SY, Chen YL, et al: Melatonin pretreatment enhances the therapeutic effects of exogenous mitochondria against hepatic ischemia-reperfusion injury in rats through suppression of mitochondrial permeability transition. J Pineal Res 61: 52-68, 2016.

29. Caldwell S: NASH Therapy: Omega 3 supplementation, vitamin $\mathrm{E}$, insulin sensitizers and statin drugs. Clin Mol Hepatol 23: 103-108, 2017. 
30. Perumpail BJ, Cholankeril R, Yoo ER, Kim D and Ahmed A: An overview of dietary interventions and strategies to optimize the management of non-alcoholic fatty liver disease. Diseases 5: 23, 2017.

31. Yang H, Ramani K, Xia M, Ko KS, Li TW, Oh P, Li J and Lu SC: Dysregulation of glutathione synthesis during cholestasis in mice: Molecular mechanisms and therapeutic implications. Hepatology 49: 1982-1991, 2009.

32. Burhans MS, Flowers MT, Harrington KR, Bond LM, Guo CA, Anderson RM and Ntambi JM: Hepatic oleate regulates adipose tissue lipogenesis and fatty acid oxidation. J Lipid Res 56: 304-318, 2015

33. Tong L: Acetyl-coenzyme A carboxylase: Crucial metabolic enzyme and attractive target for drug discovery. Cell Mol Life Sci 62: 1784-1803, 2005.

34. Easlon E, Tsang F, Skinner C, Wang C and Lin SJ: The malate-aspartate NADH shuttle components are novel metabolic longevity regulators required for calorie restriction-mediated life span extension in yeast. Genes Dev 22: 931-944, 2008.

35. Kim EY, Kim WK, Kang HJ, Kim JH, Chung SJ, Seo YS, Park SG, Lee SC and Bae KH: Acetylation of malate dehydrogenase 1 promotes adipogenic differentiation via activating its enzymatic activity. J Lipid Res 53: 1864-1876, 2012.

36. Rudraiah S, Zhang $X$ and Wang L: Nuclear receptors as therapeutic targets in liver disease: Are we there yet?. Annu Rev Pharmacol Toxicol 56: 605-626, 2016.
37. Halilbasic E, Baghdasaryan A and Trauner M: Nuclear receptors as drug targets in cholestatic liver diseases. Clin Liver Dis 17: 161-189, 2013.

38. Montagner A, Korecka A, Polizzi A, Lippi Y, Blum Y, Canlet C, Tremblay-Franco M, Gautier-Stein A, Burcelin R, Yen YC, et al: Hepatic circadian clock oscillators and nuclear receptors integrate microbiome-derived signals. Sci Rep 6: 20127, 2016.

39. Tan DX, Manchester LC, Esteban-Zubero E, Zhou Z and Reiter RJ: Melatonin as a potent and inducible endogenous antioxidant: Synthesis and metabolism. Molecules 20: 18886-18906, 2015.

40. Jaeschke H, Gores GJ, Cederbaum AI, Hinson JA, Pessayre D and Lemasters JJ: Mechanisms of hepatotoxicity. Toxicol Sci 65: 166-176, 2002

41. Semak I, Korik E, Antonova M, Wortsman J and Slominski A: Metabolism of melatonin by cytochrome P450s in rat liver mitochondria and microsomes. J Pineal Res 45: 515-523, 2008.

42. Woolbright BL and Jaeschke H: Xenobiotic and endobiotic mediated interactions between the Cytochrome P450 system and the inflammatory response in the liver. Adv Pharmacol 74: 131-161, 2015.

This work is licensed under a Creative Commons Attribution-NonCommercial-NoDerivatives 4.0 International (CC BY-NC-ND 4.0) License. 